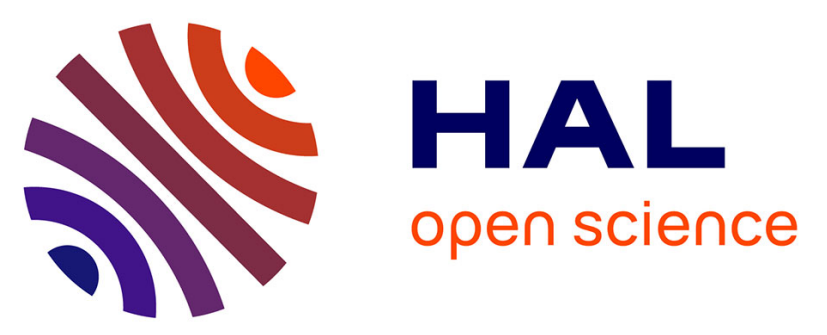

\title{
Surface Modification of CdE (E: S, Se, and Te) Nanoplatelets to Reach Thicker Nanoplatelets and Homostructures with Confinement-Induced Intraparticle Type I Energy Level Alignment
}

Nicolas Moghaddam, Corentin Dabard, Marion Dufour, Hong Po, Xiangzhen $\mathrm{Xu}$, Thomas Pons, Emmanuel Lhuillier, Sandrine Ithurria

\section{To cite this version:}

Nicolas Moghaddam, Corentin Dabard, Marion Dufour, Hong Po, Xiangzhen Xu, et al.. Surface Modification of CdE (E: S, Se, and Te) Nanoplatelets to Reach Thicker Nanoplatelets and Homostructures with Confinement-Induced Intraparticle Type I Energy Level Alignment. Journal of the American Chemical Society, In press, 10.1021/jacs.0c10336 . hal-03117326

\author{
HAL Id: hal-03117326 \\ https://hal.science/hal-03117326
}

Submitted on 21 Jan 2021

HAL is a multi-disciplinary open access archive for the deposit and dissemination of scientific research documents, whether they are published or not. The documents may come from teaching and research institutions in France or abroad, or from public or private research centers.
L'archive ouverte pluridisciplinaire HAL, est destinée au dépôt et à la diffusion de documents scientifiques de niveau recherche, publiés ou non, émanant des établissements d'enseignement et de recherche français ou étrangers, des laboratoires publics ou privés. 
Surface modification of CdE (E: S, Se and Te) nanoplatelets to reach thicker nanoplatelets and homostructures with confinement-induced intraparticle type I energy level alignment

\author{
Nicolas Moghaddam ${ }^{1}$, Corentin Dabard ${ }^{1,2}$, Marion Dufour ${ }^{1}$, Hong $\mathrm{Po}^{1}$, Xiang Zhen $\mathrm{Xu}^{1}$, Thomas Pons ${ }^{1}$, \\ Emmanuel Lhuillier ${ }^{2}$, Sandrine Ithurria ${ }^{1, *}$ \\ ${ }^{1}$ Laboratoire de Physique et d'Etude des Matériaux, ESPCI-Paris, PSL Research University, Sorbonne \\ Université UPMC Univ Paris 06, CNRS, 10 rue Vauquelin 75005 Paris, France \\ ${ }^{2}$ Sorbonne Université, CNRS, Institut des NanoSciences de Paris, INSP, F-75005 Paris, France
}

* Corresponding author: sandrine.ithurria@espci.fr

Abstract: Two-dimensional II-VI semiconductor nanoplatelets (NPLs) present exceptionally narrow optical features due to their thickness defined at the atomic scale. Since thickness drives the bandedge energy, its control is of paramount importance. Here, we demonstrate that native carboxylate ligands can be replaced by halides that partially dissolve cadmium chalcogenide NPLs at the edges. The released monomers then recrystallize on the wide top and bottom facets, leading to an increase in NPL thickness. This dissolution/recrystallization method is used to increase NPL thickness to $9 \mathrm{MLS}$ while using $3 \mathrm{ML}$ NPLs as the starting material. We also demonstrate that this method is not limited to CdSe and can be extended to CdS and CdTe to grow thick NPLs. When the metal halide precursor is introduced with a chalcogenide precursor on the NPLs, CdSe/CdSe, CdTe/CdTe, CdSe/CdTe core/shell homo- and heterostructures are achieved. Finally, when an incomplete layer is grown, NPLs with steps are synthesized. These stress-free homostructures are comparable to type I heterostructures, leading to recombination of the exciton in the thicker area of the NPLs. Following the growth of core/crown and core/shell NPLs, it affords a new degree of freedom for the growth of structured NPLs with designed band engineering, which has so far been only achievable for heteromaterial nanostructures. 


\section{INTRODUCTION}

In the last decade, II-VI 2D semiconductor nanoplatelets (NPLs) have raised intense interest due to their exceptional optical features, which are tunable due to their thickness control at the atomic scale. ${ }^{1}$ In such nanocrystals, the quantum confinement is limited to one direction. Consequently, their emission linewidths are three times narrower than in the case of spherical particles, and they present a high exciton binding energy ${ }^{2,3}$ leading to a short fluorescence lifetime. ${ }^{4}$ Among various colloidal $2 \mathrm{D}$ nanomaterials, ${ }^{5-10}$ zinc blende $2 \mathrm{D}$ cadmium chalcogenides offer the highest versatility for the growth of heterostructures. They can be doped due to partial cation exchange, with copper ${ }^{11}$ or silver ${ }^{12,13}$ leading to a high quantum yield redshifted emission. A second semiconductor can also be grown parallel to the confinement direction, resulting in core/shell NPLs, or perpendicular to it, leading to core/crown heterostructures. ${ }^{1}$ For the latter, fine engineering of the band alignment enables localization of the exciton in different areas of the NPLs as well as high quantum yield efficiency from green to red with NPLs that can even exhibit multicolour emission. ${ }^{14-19}$ Such NPLs are suitable for low threshold lasing or light concentrators. ${ }^{15,20,21}$

In the zinc blende NPLs, the thickness is oriented along the [001] direction, ${ }^{22,23}$ with an alternation of cadmium and chalcogenide planes, completed by cadmium planes on both sides ( $\mathrm{CdE}$ NPLs, with E:S, Se, Te; of N MLs present, N planes of E and N+1 planes of $\mathrm{Cd}$ ). The neutrality of the NPLs is ensured by carboxylate ligands, whose binding leads to an order of magnitude faster growth rate in the lateral direction than in the thickness direction. To overcome this passivation, three major strategies have been proposed to grow core/shell NPLs: (i) colloidal atomic layer deposition (c$A L D)^{24,25}$, (ii) decomposition at room temperature of thioacetamide with metal salts $^{26}$ and (iii) decomposition of thiolates and metal salts at high temperatures. ${ }^{27-29}$ The last method leads to the formation of core/shell NPLs with high quantum yield efficiency but is limited to the growth of cadmium and zinc sulfide and zinc selenide semiconductors. ${ }^{30,31}$ In contrast, the c-ALD method ensures layer-by-layer growth of metal sulfide and metal selenide semiconductors. However, it requires highly reactive selenide and sulfide anions that need to be handled in a glovebox to prevent their oxidation. This method can be applied to the growth of telluride-based semiconductors, but their sensitivity to air is even stronger.

Thus, there remains a lack of control concerning the growth of NPLs in terms of thickness, particularly for telluride- and selenide-based semiconductors. In early reports, the direct syntheses of CdSe NPLs were limited to thicknesses ranging from 2 to $5 \mathrm{MLs}{ }^{23}$ More recently, in 2018, Moreels' group ${ }^{32}$ and Talapin's group ${ }^{33}$ successfully synthesized $6 \mathrm{ML}$ CdSe NPLs or more by adding $\mathrm{CdCl}_{2}$ to the reaction medium. In these syntheses, the formation of thicker NPLs is allowed due to a decrease in the surface energy brought about by halide ligand joints with a kinetically limited reaction. ${ }^{32-35}$ However, obtaining a single population of NPLs remains challenging, and such growth has not yet been applied to CdTe. Halide ligands have also emerged as a way to control the shape of metallic and semiconductor nanoparticles. ${ }^{36}$ For example, the presence of chlorinated solvent enables the growth of 2D-shaped $\mathrm{PbS}$ nanoparticles ${ }^{8}$ or the control of the crystal structure of 2D-shaped CdSe nanoparticles. ${ }^{37}$ Similarly, changing the nature of the halides enables the growth of $\mathrm{HgTe}^{37}$ or $\operatorname{InP}$ nanocrystals with various sizes. ${ }^{38,39}$

In this article, we show that halide ligands can be used to increase the thickness of preexisting cadmium chalcogenide NPLs. The addition of cadmium halides in a reaction medium at mild 
temperatures $\left(\approx 160^{\circ} \mathrm{C}\right)$ leads to full ligand exchange on the NPLs, from carboxylates to halides, followed by partial dissolution, mostly by edges. The released monomers then recrystallize on the wide facets of the NPLs, leading to an increase in the thickness in steps of 2 monolayers. Thus, NPLS passivated by halide ligands can be used for the growth of thicker CdSe and CdTe homostructures with improved monodispersity as well as core/shell homo- and heterostructures. The growth of these NPLs can also be stopped by an additional ligand exchange from halides to carboxylates. In this way, NPLs with either complete or partial layers can be synthesized. In the latter, a single homostructured NPL presents steps, exhibiting areas with various thicknesses and so different levels of confinement. As a consequence, the exciton will preferably recombine in a less confined area, i.e., the thicker part of the NPL. Thus, we present the first synthesis of stress-free homostructures with a confinement-induced intraparticle type I energy level alignment. This is of particular interest considering that type I band alignments are commonly obtained in heterostructures of semiconductors for which crystal structure and lattice mismatch need to be considered. ${ }^{40}$. This growth offers a new degree of freedom in the synthesis of structured nanoparticles.

\section{RESULTS AND DISCUSSION}

1. Dissolution and recrystallization

In this sections, we first show that in the presence of halide ligands, without chalcogenide precursors, NPLS are partially dissolved, mostly at the edges, and recrystallize onto wide facets, leading to thicker NPLs. Three ML (resp. $4 \mathrm{ML}$ ) CdSe NPLs are used as the starting materials for these experiments. Consequently, they are first synthesized with well-established procedures. ${ }^{23,41}$ These NPLs present their first excitonic peaks at $462 \mathrm{~nm}$ (resp. $512 \mathrm{~nm}$ ) and present lateral dimensions ranging from $100 \mathrm{~nm}$ to $500 \mathrm{~nm}$ (resp. from $10 \mathrm{~nm}$ to $50 \mathrm{~nm}$ ). After purification, the NPLs are suspended in ODE in the presence of oleic acid (the amount of oleic acid introduced has little effect on the reaction rate (see Supp Info figure S1), once it is present), without chalcogens. The solution is then heated at approximately $160^{\circ} \mathrm{C}$ - the temperature of injection for a metal halide/phosphine complex. ${ }^{42,43}$ In 2019, Dufour et al. showed that NPLs can be passivated by halides and stabilized by amines through hydrogen bonds. ${ }^{44}$ Here, we surmise that the hydrogens from carboxylic acid can play the same role, leading to colloidal stability, without competing with halides for surface binding. The phosphines have a dual effect. They are used to complex the metal halide to obtain a soluble precursor in the reaction medium. ${ }^{42,43}$ They also participate in NPL stabilization, as revealed by Energy-dispersive $X$-ray spectroscopy (see Supp Info figure S2). We also noticed that tricotylphosphine (TOP), with its longer aliphatic chains, enabled us to obtain a clearer reaction medium for small laterally sized NPLs thanks to an increased steric hindrance between NPLs.

The different steps of the reactions were followed by the application of absorption spectroscopy (see Figure 1 (a.) and (f.)). The introduction of the metal halide/phosphine is immediately followed by a redshift of the optical features from $462 \mathrm{~nm}$ to $485 \mathrm{~nm}$ (resp. from 512 $\mathrm{nm}$ to $530 \mathrm{~nm}$ ) for the $3 \mathrm{ML}$ CdSe NPLs (resp. $4 \mathrm{ML}$ CdSe), which is attributed to ligand exchange from carboxylates to halide ligands. ${ }^{44}$ In Figure 1 (a.), at $30 \mathrm{~s}$, the absorption spectrum still exhibits two transitions attributed to the heavy hole to electron (hh-e) and light hole to electron (lh-e) transitions in $3 \mathrm{ML}$ CdSe NPLs. Over time, two additional redshifted transitions appear at approximately $550 \mathrm{~nm}$ and $520 \mathrm{~nm}$. Finally, after 7 minutes, the transitions from the $3 \mathrm{ML}$ CdSe NPLs have completely 
disappeared, while two absorption peaks at $560 \mathrm{~nm}$ and $520 \mathrm{~nm}$, attributed to $5 \mathrm{ML}$ CdSe NPLs passivated by halide ligands, are now visible. Then, the reaction continues, with an additional loss of the excitonic transitions followed by a recovery of new absorption features at approximately $610 \mathrm{~nm}$ originating from $7 \mathrm{ML}$ CdSe NPLs. ${ }^{32,41}$ Finally, after 115 minutes, the absorption reaches $645 \mathrm{~nm}$, corresponding to $9 \mathrm{ML} \mathrm{CdSe} \mathrm{NPLs.}{ }^{41}$ In this reaction, the broadening of the absorption peaks is attributed to a mix or defects of ligands on the NPLs. In such highly confined particles, a slight modification in the confined direction, the thickness, including the surface ligands, leads to a broadening of the optical features. The TEM images reveal conservation of the 2D shape but with less defined edge facets than before and the appearance of holes on extended NPLs (see Figure 1 (b.) to (e.) and also Supp Info Figure S3 for less extended NPLs). For thick NPLs, heterogeneous contrasts extended over a few hundred $\mathrm{nm}^{2}$ - also appear on a single NPL. They most likely arise due to inhomogeneous surface chemistry. Indeed, if this contrast arises from inhomogeneities in the core thicknesses, such large areas would affect the optical properties with the absorption signature of thicker or thinner NPLs. In the case of 4 ML CdSe NPLs (see Figure 1 (f.)), comparable behaviours are observed. After fast ligand exchange, excitonic transitions at $585 \mathrm{~nm}$ and $550 \mathrm{~nm}$ are recovered at 10 minutes, corresponding to $6 \mathrm{ML}$ CdSe NPLs. The $8 \mathrm{ML}$ CdSe NPLs are obtained after 50 minutes of reaction. Again, the $2 \mathrm{D}$ shape of the NPLs is preserved. Here, a reduction in their lateral dimensions (mostly the length) is observed in TEM images (see Figure 1 (g.) to (i.)). An additional experiment with an absorption probe placed directly inside the reaction flask shows an isosbestic point at $426 \mathrm{~nm}$. Thus, the volume of crystalline CdSe remains constant throughout the reaction (see Supp Info Figure S4). All these observations suggest a dissolution/recrystallization process, which induces a reduction in the lateral dimensions as the thickness of the NPLs increases.
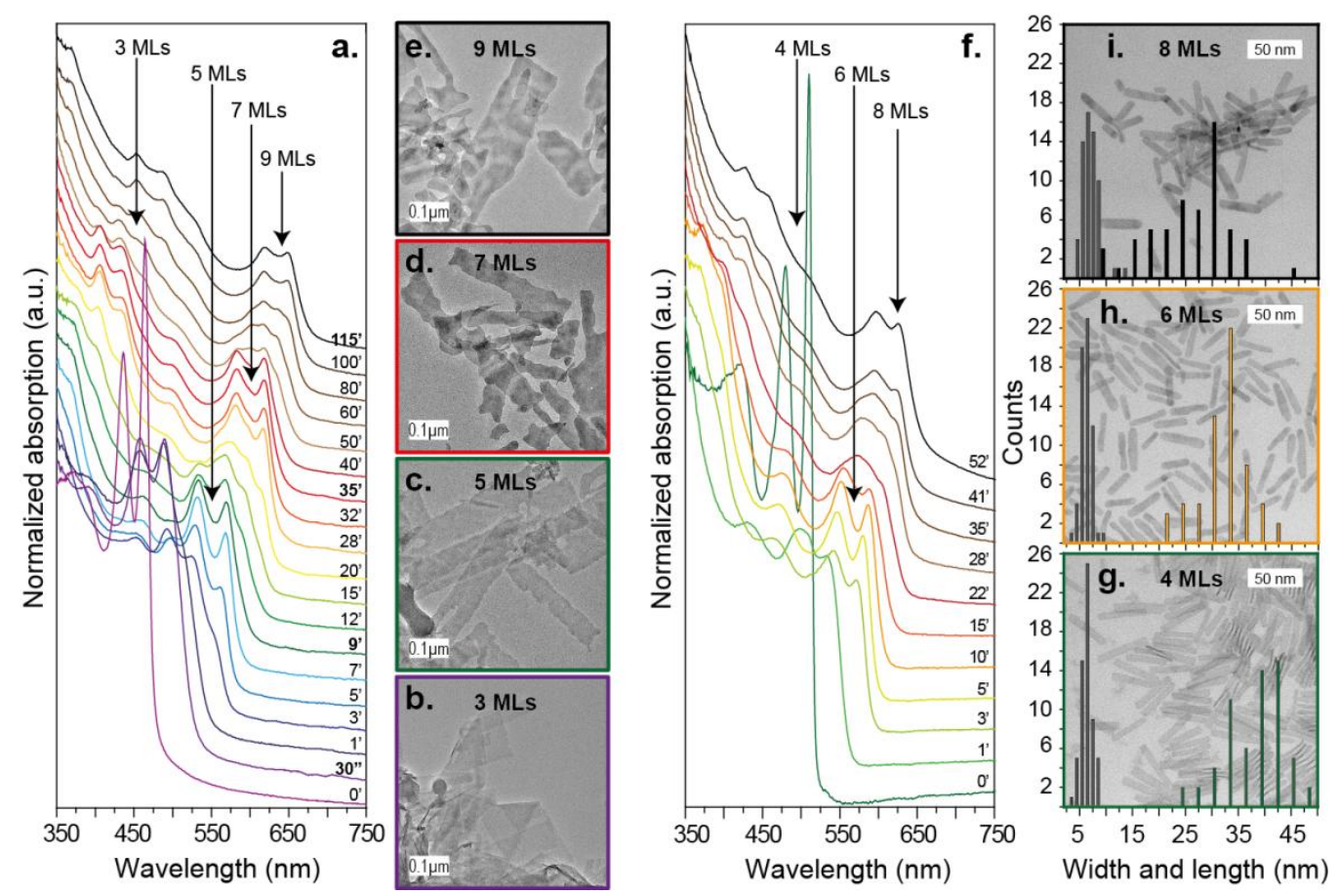

Figure 1 : (a.) Absorption spectra for aliquots taken during the reaction at $160^{\circ} \mathrm{C}$. It shows the transformation from $3 \mathrm{ML}$ to $5 \mathrm{ML}$, then to $7 \mathrm{ML}$ and finally to $9 \mathrm{ML}$ CdSe NPLs. The spectra have been normalized to $350 \mathrm{~nm}$.

(b.), (c.), (d.) and (e.) TEM images of 3, 5, 7 and 9 ML CdSe NPLs. (f.) Absorption spectra for aliquots taken during the reaction at $160^{\circ} \mathrm{C}$ (the spectra are normalized to $350 \mathrm{~nm}$.). This shows the transformation from $4 \mathrm{ML}$ to 6 ML and finally to 8 ML CdSe NPLs. (g.), (h.) and (i.) TEM images and the length and width distributions for 4, 
In this reaction, the first step consists of ligand exchange from carboxylates to halides. If these carboxylates are in excess compared to halides, they compete for binding on the $<001>$ facets of the NPLs, leading to an inefficient reaction (see Supp Info Figure S5). Consequently, the carboxylate/halide ratio determines the surface energy and reactivity of the NPLs. This is why Moreels and coworkers had to conduct the growth of the NPLs at $300^{\circ} \mathrm{C}$ to compensate for the high $\mathrm{Cd}(\text { carboxylates })_{2}: \mathrm{CdCl}_{2}$ ratio. ${ }^{32}$

In a second step, after ligand exchange, a slow redshift of the optical features is observed, indicating a modification of the confinement, and, hence, a growth in the thickness. Since no extra chalcogenide precursors were introduced in the reaction medium, this growth originates from a partial dissolution of the NPLs followed by recrystallization on the top and bottom facets. These two identical facets react the same way independently, which explains the increase in the NPL thickness by $2 \mathrm{MLs}$. It should be noted that despite the growth of the top and bottom wide facets at the same rate, a single NPL can present areas of $\mathrm{N}, \mathrm{N}+1$ and $\mathrm{N}+2 \mathrm{MLs}$ during growth if these two opposite facets have nucleated at different times (see Part 3). Finally, using the same reasoning as in the first step, an excess of $\mathrm{Cd}$ (acetate) ${ }_{2}$ can be added, increasing the carboxylate/halide ratio to deactivate the surface and stop the dissolution/recrystallization reaction. This $\mathrm{Cd}$ (acetate) ${ }_{2}$ addition also induces a rearrangement of the surface. This leads, first, to a blueshift of the optical features in the absorbance followed by a resurgence of the excitonic peaks (see Supp Info Figures S8, S10 and S11).

According to Norris's group, CdSe monomer desorption, mostly by the edges, strongly depends on the thickness of the NPL and occurs mostly via the edges. ${ }^{45}$ In contrast, the recrystallization step, if it occurs on the wide top and bottom facets, should not depend on the NPL thickness ${ }^{45}$. In the model proposed by the same group, the energy barrier to island nucleation strongly depends on both the surface energy and supersaturation. The introduction of chloride in the mixture decreases the surface energy but also leads to an increase in supersaturation. Hence, the kinetic barrier to nucleation of a new layer on a wide facet decreases. The islands most likely nucleate at the edges or in the corners of the wide facets where the binding energy of the ligands decreases. ${ }^{46}$ As a consequence, an island can be nucleated on a wide facet and can extend from the edge to the centre. Lateral extension can jointly occur; however, thicker NPLs can only be obtained if an island on a wide facet has already nucleated. In that case, starting from N ML NPLs, N+1 ML NPLs should grow much faster than N+2 ML NPLs, and a mix of NPLs should be obtained. In Figure 1, growth occurs in steps of $2 \mathrm{MLs}$, which argues in favour of recrystallization mainly by wide facets regardless of the NPL thickness.

These observations slightly differ from those of the group of Moreels and coworkers. In their work, they also showed a constant volume of CdSe in the reaction medium while switching from $\mathrm{N}$ ML CdSe NPLs to thicker NPLs but with an increase in the NPL lateral dimensions. In their experiments, the partial ligand exchange from carboxylates to halides, brought about by a high $\mathrm{Cd}$ (carboxylates) $)_{2}: \mathrm{CdCl}_{2}$ ratio, led to a decrease in the surface energy and probably the formation of small islands. However, due to ligand competition, these islands will not extend efficiently, and Oswald ripening between NPLs of various thicknesses can possibly occur, as described by Norris and coworkers. This can explain the increase in the lateral dimension with the increased thickness, as well as the increase in the thickness due to one monolayer instead of two.

To verify this mechanism, we proposed (see Figure 2 (a.)), experiments with constant amounts of precursors that are performed at various temperatures. The time necessary to increase 
the thickness by 2 and 4 monolayers starting from 3 and 4 ML CdSe NPLs was monitored. Initially, the $3 \mathrm{n}$ moles of Se in $3 \mathrm{ML}$ (resp. $4 \mathrm{n}$ moles of Se in the $4 \mathrm{ML}$ ) CdSe NPLs are distributed over 5 (resp. 6) planes for the $5 \mathrm{ML}$ (resp. $6 \mathrm{ML}$ ) CdSe NPLs (i.e., 3n/5 (resp 4n/6) moles per planes). Therefore, the total surface area of the NPLs should be reduced by $40 \%(3 n-3 n / 5)$ (resp. 33\% (4n-4n/6)) for the switch from $3 \mathrm{ML}$ to $5 \mathrm{ML}$ (resp. $4 \mathrm{ML}$ to $6 \mathrm{ML}$ ) CdSe NPLs. In other words $40 \%$ (resp. 33\%) of the total volume of the $3 \mathrm{ML}$ (resp. $4 \mathrm{ML}$ ) CdSe NPLs dissolve and recrystallize on these $3 \mathrm{ML}$ (resp. $4 \mathrm{ML}$ ) CdSe NPLs to obtain the $5 \mathrm{ML}$ (resp. $6 \mathrm{ML}$ ) CdSe NPLs. Continuing the reaction, 28\% (resp. 25\%) of the $5 \mathrm{ML}$ (resp. $6 \mathrm{ML}$ ) CdSe NPLs are dissolved and recrystallized to obtain $7 \mathrm{ML}$ (resp. $8 \mathrm{ML}$ ) CdSe NPLs (see Figure 2 (b.)). In total, $57 \%$ (resp. 50\%) of $3 \mathrm{ML}$ (resp. $4 \mathrm{ML}$ ) CdSe NPLs need to be dissolved to obtain $7 \mathrm{ML}$ (resp. $8 \mathrm{ML}$ ) CdSe NPLs. For the case of $4 \mathrm{ML}$ CdSe NPLs, there is indeed a decrease in the lateral dimensions, which is not as large as expected. On average, the 4, 6 and $8 \mathrm{ML}$ CdSe NPLs have lateral dimensions of $38 \pm 2 \mathrm{~nm} \times 6 \pm 0.5 \mathrm{~nm}, 32 \pm 2 \mathrm{~nm} \times 5,5 \pm 0.5 \mathrm{~nm}$ and $26 \pm 5 \mathrm{~nm} \times 6,5 \pm 1 \mathrm{~nm}$ and a surface area of $228 \pm 31 \mathrm{~nm}^{2}, 176 \pm 27 \mathrm{~nm}^{2}$ and $169 \pm 52 \mathrm{~nm}^{2}$, respectively(see Figure 1 (g), (h.) and (i.)). Therefore, as the thickness increases, the lateral dimensions decrease, but the polydispersity in the lateral dimension also increases. Indeed, the NPLs with the smallest lateral dimensions dissolve faster. Thus, as the NPLs grow in thickness, lateral dimension defocusing also occurs.

Figure 2 (c.) represents the inverse of time necessary to increase the thickness from $3 \mathrm{MLs}$ to $5 \mathrm{MLs}$ (resp. $4 \mathrm{MLs}$ to $6 \mathrm{MLs}$ ), from 3 to $7 \mathrm{MLs}$ (resp. $4 \mathrm{MLs}$ to $8 \mathrm{MLs}$ ) and from 5 to $7 \mathrm{MLs}$ (resp. 6 MLs to $8 \mathrm{MLs}$ ) multiplied by the inverse of the temperature as a function of the inverse of the temperature. These points were normalized to the ratio of CdSe NPLs $\left(x_{s}\right)$ that had been dissolved and recrystallized. This enables the use of the Eyring equation to determine the enthalpy and entropy of the transition state of the reaction involving the dissolution and recrystallization of CdSe monomers. ${ }^{47,48}$ The constant rate $k$ for a chemical reaction is written as follows:

$$
k=\frac{\kappa k_{B} T}{h} e^{-\frac{\Delta^{\ddagger} G}{R T}}=\frac{\kappa k_{B} T}{h} e^{\frac{\Delta^{\ddagger} S}{R}} e^{-\frac{\Delta^{\ddagger} H}{R T}}
$$

where $k_{B}$ is the Boltzmann constant, $h$ is Planck's constant, $\Delta^{\neq} H$ and $\Delta^{\neq} S$ are the enthalpy and entropy of activation, respectively, $T$ is the temperature of the reaction and $K$ is a transmission coefficient.

a. $\mathrm{m}_{\mathrm{S}}[\mathrm{CdSe}]_{\mathrm{N}}+2 \mathrm{Cl} \stackrel{(1)}{\longrightarrow} \mathrm{m}_{\mathrm{s}}\left(1-\mathrm{x}_{\mathrm{S}}\right)[\mathrm{CdSe}]_{\mathrm{N}}+\mathrm{x}_{\mathrm{S}} \mathrm{CdCl}_{2}+\mathrm{x}_{\mathrm{S}} \mathrm{Se}^{2-}$

limiting process

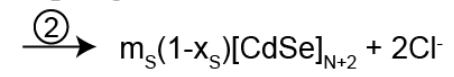

b.
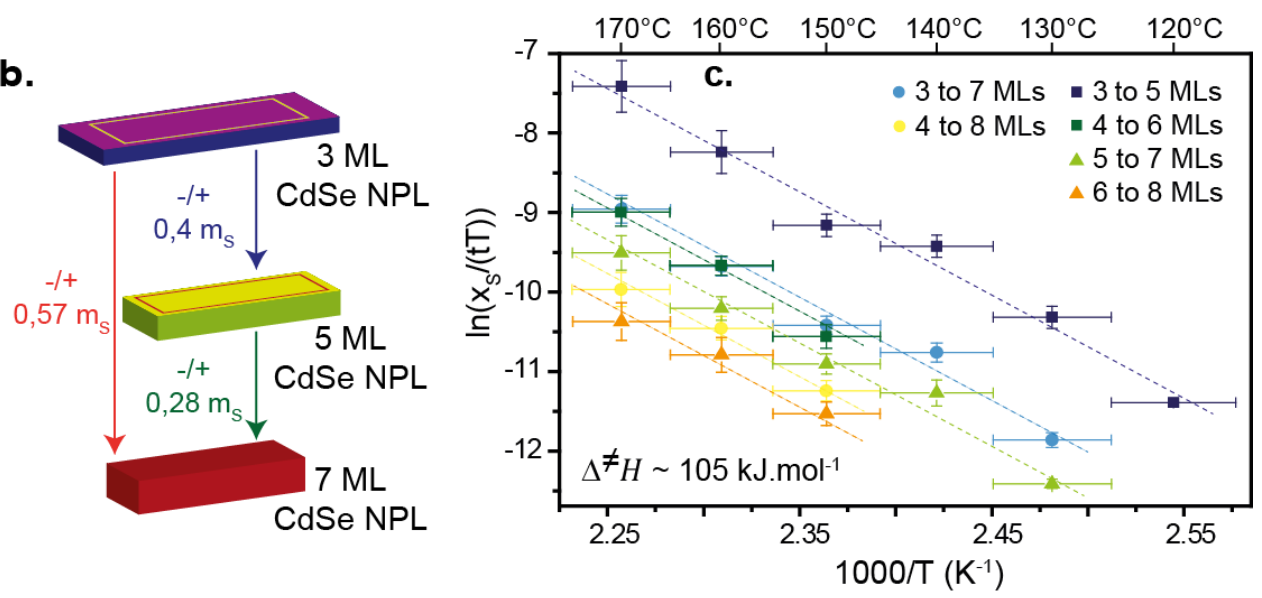
Figure 2 : (a.) A two-step reaction leads to the growth of the NPLs in thickness with first dissolution of the NPLS (1) followed by recrystallization of the monomers on the wide facets (2). $N$ represents the number of layers in the NPLs, $m_{s}$ is the molar quantity of the surface and $x_{s}$ is the dissolved ratio. The first step is the limiting process (b.) Scheme showing the dissolution and recrystallization of NPLS. The amount of dissolved NPLs depends on the thickness of the starting NPLs. The thinner the NPLs are, the more the NPLs are dissolved. Forty percent of $3 \mathrm{ML}$

CdSe NPLs need to be dissolved to obtain 5 ML CdSe NPLs, and 28\% of these 5 ML CdSe NPLs need to be dissolved to obtain $7 \mathrm{ML}$ CdSe NPLs. (c.) Eyring plot for the dissolution/recrystallization process starting from 3 $M L$ and $4 M L C d S e N P L s$.

In Figure 2(c.), the linear trend enables us to determine an enthalpy of activation of $\approx 105$ $\mathrm{kJ}$. $\mathrm{mol}^{-1}$ for the switch from 3 to $5 \mathrm{ML} \mathrm{CdSe} \mathrm{NPLs}$ and from 5 to $7 \mathrm{ML} \mathrm{CdSe} \mathrm{NPLs.} \mathrm{A} \mathrm{similar} \mathrm{value} \mathrm{was}$ found for the switch from 4 to $6 \mathrm{ML}$ and 6 to $8 \mathrm{MLCdSe}$ NPLs. Thus, the enthalpy of activation is constant regardless of the starting and final NPL thickness. On the other hand, the intercept of the curve strongly depends on NPL thickness, despite the normalization to $\mathrm{x}_{\mathrm{s}}$. In other words, the rate of the reaction decreases with increasing thickness, which is in good agreement with the model proposed by Norris and coworkers. Here, we estimate that the dissolution of the $3 \mathrm{ML}$ (resp. $4 \mathrm{ML}$ ) CdSe NPLs is $\approx 6$ times (resp. $\approx 4$ times) faster than the dissolution of the $5 \mathrm{ML}$ (resp. $6 \mathrm{ML}$ ) CdSe NPLs. Thus, in the two-step process implying dissolution followed by recrystallization, dissolution is indeed the limiting process. In addition, we have shown that the released monomers react equally on NPLs of different thicknesses (see Supp Info Figure S6).

Once dissociated from the edges, the monomers can react with both wide and narrow facets; however, this reaction occurs with a high probability on the wide facets since they represent the largest percentage of the available surface on the lateral facets, competition between dissolution and recrystallization occurs. With enough $\mathrm{CdCl}_{2}$ precursors in the mixture, the system is under kinetic control, and the reaction is not limited by the deposition of monomers on the surface. Thus, if the dissociation rate is sufficient, 1 layer on the top and bottom wide facets can be fully grown. Conversely, if the dissociation rate is too low (at low temperature or with a low amount of $\mathrm{CdCl}_{2}$ precursors or for thick NPLs), NPLs with partial layers are obtained, meaning that NPLs can present steps. Here, a 2-layer by 2-layer growth can be controlled through the reaction rate, which is increased by the amount of $\mathrm{CdCl}_{2}$ introduced and the temperature (see Supp Info Figure S7). Consequently, if the switch from $\mathrm{N}$ to $\mathrm{N}+2 \mathrm{ML} N \mathrm{NLs}$ is too slow (slow dissolution is favoured in thick NPLs), the growth is no longer limited by the kinetics, and multi-stepped NPLs will be obtained, which broadens the thickness distribution. Hence, to maintain a low thickness polydispersity, either additional halide ligands or higher temperatures are necessary. With this dissolation/recrystallization process, followed by quenching with $\mathrm{Cd}$ (acetate) ${ }_{2}$, pure populations of $5 \mathrm{ML}, 6 \mathrm{ML}$ and $7 \mathrm{ML} \mathrm{CdSe}$ NPLs were synthesized (see Supp Info Figures S11, S8 and S10). The fluorescence quantum yield (QY) for 5 and $7 \mathrm{ML}$ CdSe NPLs remains low due to their large lateral extension. In contrast, the QY for the $6 \mathrm{ML}$ CdSe NPLs reached $15 \%$ and could be further improved to $53 \%$ when passivated by $\mathrm{Br}$-OLAm ${ }^{44}$ (see Supp Info Figure S9).

We have also investigated this process through the scope of other cadmium halide precursors and other cadmium chalcogenide NPLs (see Supp Info Figure S6 and Figure 3 (a.) and (d.)). The kinetic rate decreases with the halide size. Thus, for CdSe NPLs, the temperature needs to be increased by $25^{\circ} \mathrm{C}$ to have an equivalent kinetic rate while changing cadmium chloride by cadmium bromide. The kinetic rate was even lower for $\mathrm{Cdl}_{2}$ (see Supp Info Figure S11). This behaviour has 
already been observed by Lesnyak and coworkers and is attributed to the increase in surface energy as a function of the halide size. ${ }^{34}$ In addition, the steric hindrance brought about by the halides also increases with the halide size, which can obstruct the growth in the thickness. Similarly, the chalcogen also affects the reaction rate. The negative volume energy is lower in CdS than in CdSe, itself lower than in CdTe. ${ }^{35}$ Hence, the dissolution step is less favourable in CdS than in CdSe and CdTe. That is why $\mathrm{CdCl}_{2}$ /TBP (resp. $\mathrm{CdBr}_{2} /$ TBP) was used for CdS (resp. CdTe) with a temperature of $180^{\circ} \mathrm{C}$ (resp. $160^{\circ} \mathrm{C}$ ) (See Figure 3). It should be noted that $\mathrm{CdF}_{2}$ was not considered due to its low solubility in phosphines. For the case of CdTe NPLs, the precursor was added at room temperature before heating the mixture to prevent the direct dissolution of CdTe NPLs due to the temperature. In Figure 3 (a.) and (d.), the absorption spectra exhibit a direct redshift of the optical features after the introduction of the halide ligands, followed by a continuous redshift of the absorption due to growth in the confined direction. The addition of $\mathrm{Cd}$ (acetate) ${ }_{2}$ leads to fast ligand exchange from halides to carboxylates, which decreases the kinetic rate. In this way, $5 \mathrm{ML}$ CdTe NPLs were synthesized from 3 ML CdTe NPLs and 6 ML CdS NPLs from 4 ML CdS NPLs. Their quantum yields were too low to be measured. Before that, only mixtures of thick CdTe NPLs with various thicknesses were obtained. ${ }^{23,49}$ The NPLs preserve their 2D shapes with a small degradation of their edges observed even if some holes appear inside the NPLs (see Figure 3 (c.)).
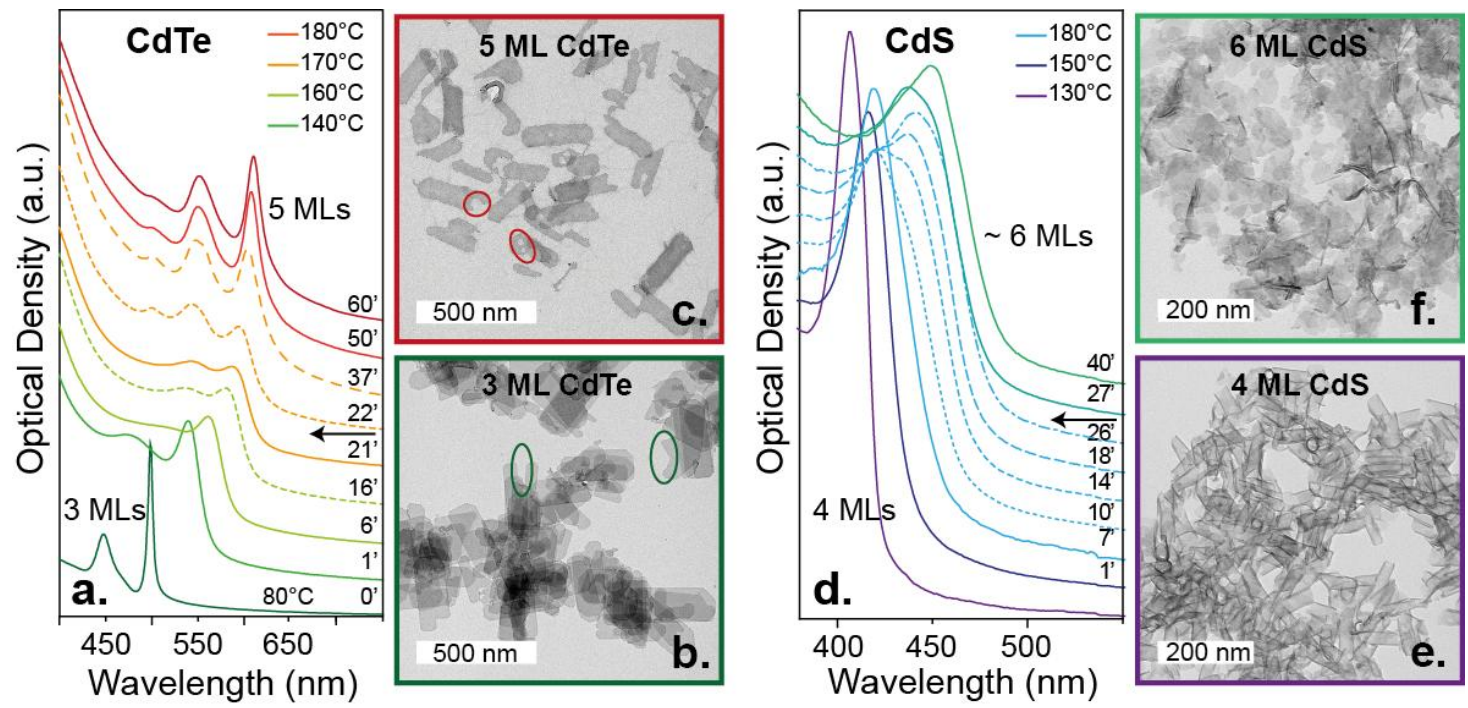

Figure 3 : (a.) Absorption spectra during the growth from $3 \mathrm{ML}$ to $5 \mathrm{ML}$ CdTe NPLs. The black arrow indicates the introduction of Cd(acetate) ${ }_{2}$. (b.) and (c.) TEM images of $3 \mathrm{ML}$ and $5 \mathrm{MLCdTe}$ NPLs. The ovals represent defects in the NPLs. (d.) Absorption spectra during the growth from 4 ML to 6 ML CdS NPLs. The black arrow indicates the introduction of Cd(acetate $)_{2}$. (f.) and (e.) TEM images of $4 \mathrm{ML}$ and $6 \mathrm{MLCdS} N \mathrm{NLS}$.

\section{Direct growth of homo and heterostructures}

In this section, we show the direct growth of a CdSe, CdTe or ZnSe shells on NPLs passivated by halide ligands. Indeed, since recrystallization is not the limiting step, halide-capped NPL surfaces can react with monomers already present in solution. Thus, this time, a chalcogenide precursor is jointly added with the metal halide/phosphine complex. Working at temperatures between $150^{\circ} \mathrm{C}$ and $170^{\circ} \mathrm{C}$, the metal halide and the chalcogenide are introduced in a 1:1 stoichiometry. In addition, NPL: $\mathrm{CdCl}_{2}$ is reduced by a factor of two compared to previous experiments to prevent the dissolution of NPLs. As in the previous section, the introduction of $\mathrm{CdCl}_{2} / \mathrm{TBP}$, here jointly with TOPSe, leads to a decrease in the NPL surface energy and an increase in supersaturation. Therefore, lateral extension is 
not more favoured than the growth in thickness. The growths were carried out on extended $3 \mathrm{ML}$ CdSe. In Figure 4 (a.), the absorption spectra taken for samples during the reaction again show a redshift of the optical features due to ligand exchange, followed by a redshift of the optical features while the reaction occurs. As soon as a partial or a full layer is grown, typically in 5 to 15 minutes, an excess of $\mathrm{Cd}$ (acetate $)_{2}$ is introduced. This enables the completion and freezing of the reaction over the thickness. In addition, the time required to grow a $1 \mathrm{ML}$ shell is independent of the NPL thickness (see Figure 4 (a.), (b.) and (c.)). The absorption features evolve until the completion of the layer (see Figure 4 (a.)). Finally, the absorption spectrum of the obtained $5 \mathrm{ML}$ CdSe NPLs exhibits sharp optical transitions at $552 \mathrm{~nm}$ and $515 \mathrm{~nm}$, as expected.
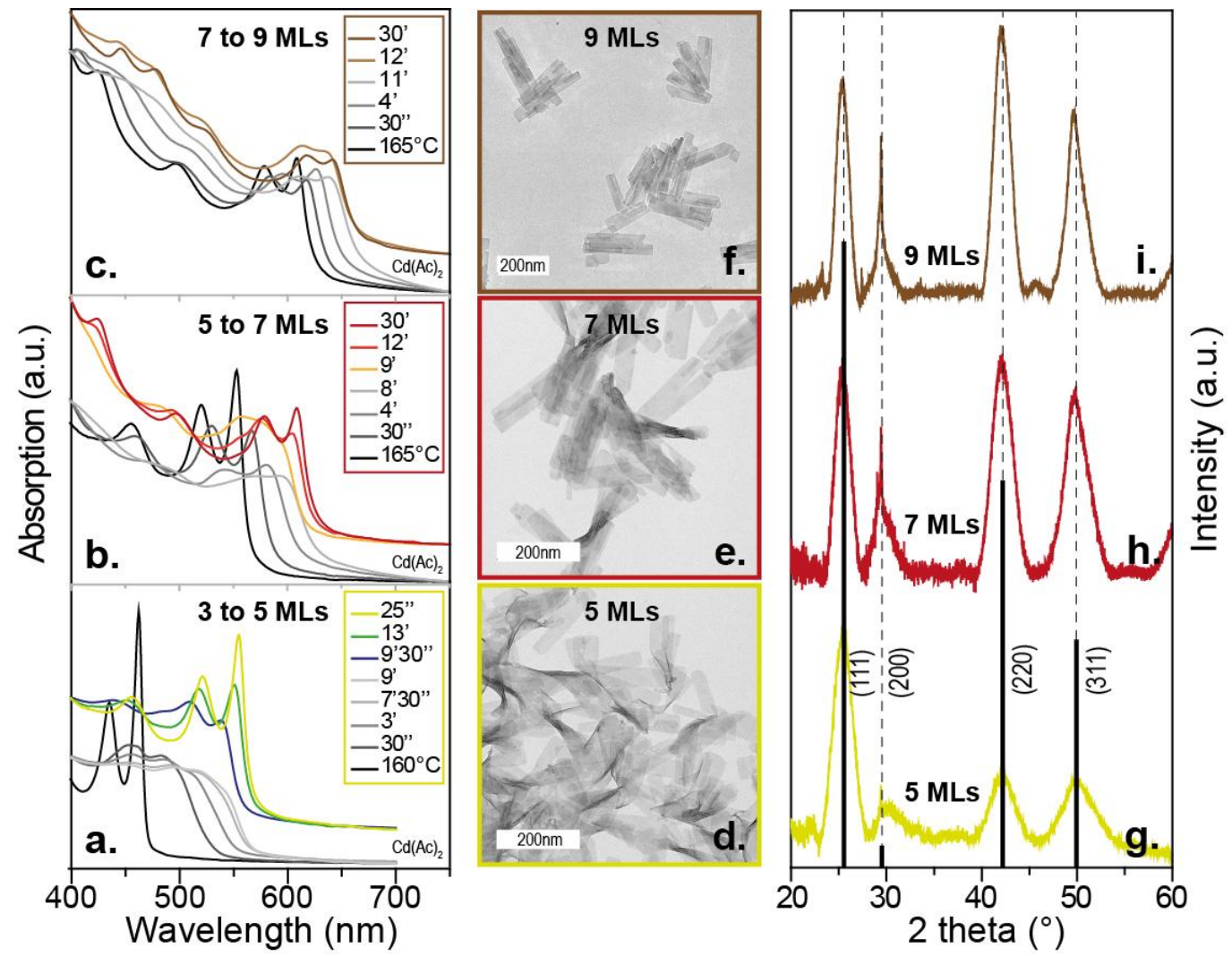

Figure 4 : (a.), (b.) and (c.) Absorption spectra measured during the growth of CdSe NPLs from $3 \mathrm{ML}$ to $5 \mathrm{ML}$, from $5 \mathrm{ML}$ to $7 \mathrm{ML}$ and from $7 \mathrm{ML}$ to $9 \mathrm{ML}$. The black and grey spectra are taken before and after the addition of a mix of $\mathrm{CdCl}_{2}$ in TBP and TOPSe, and the coloured spectra are taken after the addition of Cd(acetate) ${ }_{2}$. (d.), (e.) and (f.) TEM images of $5 \mathrm{ML}, 7 \mathrm{ML}$ and $9 \mathrm{MLCdSe} N P L s$. (g.), (h.) and (i.) XRD diagrams for the $5 \mathrm{ML}, 7 \mathrm{ML}$ and $9 \mathrm{ML} C d S$ S NPLs. The NPLs preserve their zinc blende crystal structure. The lines represent the positions of the peaks for zinc blende CdSe with a lattice parameter of $6.05 \AA$ (PDF 03-065-2891).

Concerning the emission from samples taken during the reaction, we note a low efficiency that increases over time (see Figure 5 (a.)); this change is also visible in the absorption (see Supp Info Figure S12 for comparison). Indeed, the equilibrium between halide and carboxylate ligands, initially present on the NPLs, leads to better passivation of the surface atoms and an increase in the emission efficiency. Figure 5 (a.) shows the absorption, emission and photoluminescence excitation spectra measured for the aliquots 2 days after the experiment. All the samples, from 2 to 9 minutes, show an emission redshift compared to the absorption. These emissions, despite their broadening, originate from the NPLs since the photoluminescence excitation spectra centred at different wavelengths fit the absorption spectra. At $9 \mathrm{~m} \mathrm{30}$, just after the addition of $\mathrm{Cd}$ (acetate) ${ }_{2}, 4$ excitonic peaks are 
distinguishable in the absorption spectrum at approximately $430 \mathrm{~nm}, 470 \mathrm{~nm}, 510 \mathrm{~nm}$ and $540 \mathrm{~nm}$. These peaks are attributed to the lh-e transition in $3 \mathrm{MLCdSe}$, to a mix of the hh-e and Ih-e transitions in $3 \mathrm{ML}$ and $4 \mathrm{ML}$ CdSe NPLs, to a mix of the hh-e and lh-e transitions in $4 \mathrm{ML}$ and $5 \mathrm{ML}$ CdSe NPLs and to the hh-e transitions in 5 ML CdSe NPLs, respectively. Thus, multiple thicknesses are present, but the emission solely shows one peak centred at $540 \mathrm{~nm}$. These observations are attributed to a homostructured NPL presenting steps in which the exciton will recombine in the thicker part of the NPL (see Figure 5 (b)). This will be further discussed in the last section. Finally, after 25 minutes, only $5 \mathrm{ML}$ CdSe NPLs are obtained with their first absorption transition at $550 \mathrm{~nm}$ since they are passivated by carboxylates. The linewidth is approximately $15 \mathrm{~nm}$, and it is slightly broadened to the red. The typical fluorescence quantum yield ranges from $1 \%$ to $2 \%$, in the same range as for the $3 \mathrm{ML}$ CdSe NPLS.
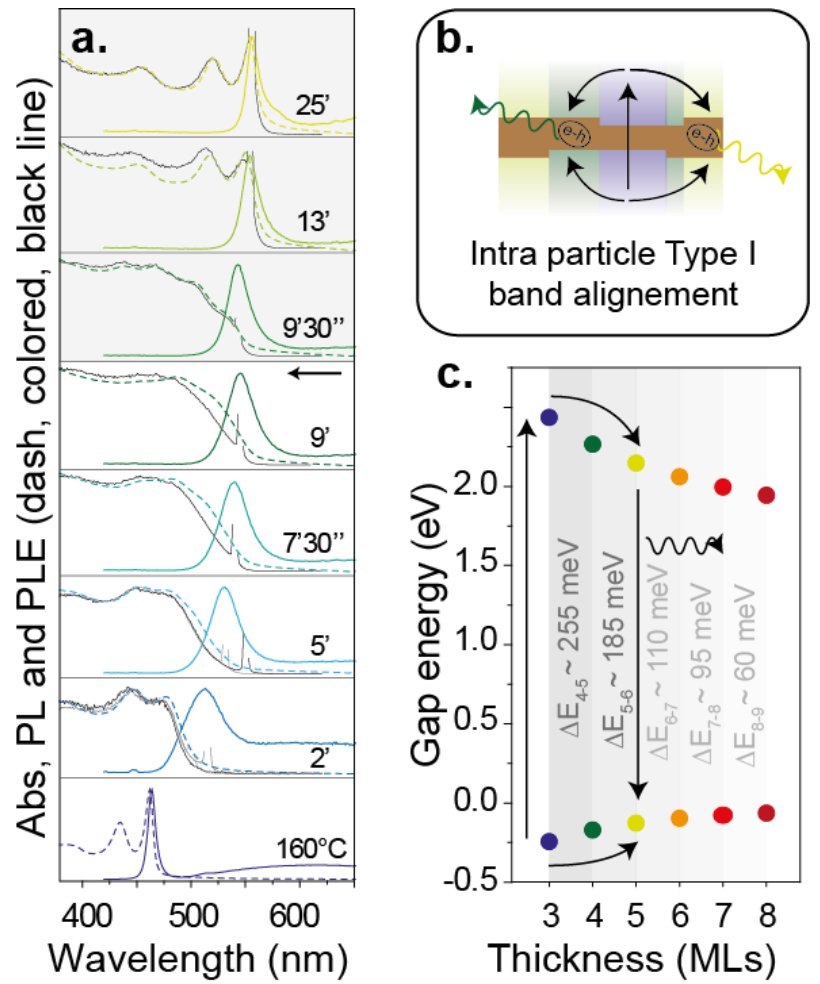

Figure 5: (a.) Absorption, photoluminescence intensity and photoluminescence excitation spectra for samples taken during the growth of a layer of CdSe on $3 \mathrm{MLCdSe} N \mathrm{NLS}$. The arrow indicates the addition of Cd(Acetate) ${ }_{2}$. The spectra were obtained 2 days after the experiment. The emission is redshifted even for partial layer growth. (b.) Scheme of the emission originating from an NPL with confinement-induced intraparticle type I energy level alignment. The exciton will recombine in the thicker part of the NPL. (c.) Band gaps of CdSe NPLs as a function of the thickness. The band alignment was proposed in the following reference. ${ }^{13}$

Additional layers can be grown in a second step after purification of the NPLs. Therefore, large lateral dimension NPLs composed of 5, 7 and 9 MLs were synthesized from $3 \mathrm{ML}$ CdSe NPLs. The shape of the NPLs is no longer degraded, the edges are straight, and no holes are visible (see Figure 4 (d.), (e.) and (f.)). Here, to ensure direct growth of the shell and not dissolution followed by recrystallization, two experiments with the same amount of NPLs were performed with and without TOPSe. At the end of the reaction, absorption spectra taken under the same conditions show that the total volume of $5 \mathrm{ML}$ CdSe NPLs was close to being twice as high in the experiment with TOPSe compared to the case without TOPSe. The total volume of initial NPLs encounters 7 atomic planes. They are further grown up to 11 atomic planes when $\mathrm{CdCl}_{2} /$ TBP and TOPSe is introduced, while these 
7 atomic planes are transformed into 11 atomic planes when only $\mathrm{CdCl}_{2} / \mathrm{TBP}$ is introduced. Therefore, in theory, the absorption ratio should be $7 / 11$, a value coherent with the experiment (see Supp Info Figure S13). In addition, the XRD diagrams show that the NPLs preserve their zinc blende structure (see Figure 4 (g.), (h.) and (i.)). The observed variation in intensity of the different peaks while increasing the thickness may be due to the orientation of the NPLs on the substrate. Indeed, as the thickness increases, the NPLs are less flexible and lie flat on the substrate, entailing an increase in the (220) intensity for thicker NPLs.

In comparison with the c-ALD method, the precursors used here are more stable, less sensitive to oxidation and do not require handling in a glovebox. Thus, these direct growth syntheses open up new possibilities for the growth of homo- and heterostructures. We indeed realized the synthesis of other homo- and heterostructures such as $\mathrm{CdSe} / \mathrm{CdTe}, \mathrm{CdTe} / \mathrm{CdTe}$ and CdSe/ZnSe (see Supp Info S14 to S16).

3. Homostructures with confinement-induced intraparticle type I energy level alignment

In this final section, we focus on the optical properties of NPLs with partial layers. Here, we intentionally synthesized NPLs with steps in their thickness by introducing $\mathrm{Cd}$ (acetate) ${ }_{2}$ during growth before the completion of a layer. For the $3 \mathrm{ML} C d S e$ NPLs, a small amount of $\mathrm{CdCl}_{2} / \mathrm{TBP}$ was introduced to nucleate only a few islands on the NPLs before the addition of $\mathrm{Cd}$ (acetate) $)_{2}$. For the second sample (Figure $6\left(\mathrm{~b}\right.$.)), a mixture of $\mathrm{CdCl}_{2} / \mathrm{TBP}$ and TOPSe was used, and $\mathrm{Cd}\left(\right.$ acetate) ${ }_{2}$ was introduced during the growth of CdSe NPLs from 6 to $8 \mathrm{MLs}$. In comparison with the experiments presented in Figure $5 \mathrm{a}$, the whole surface of the NPL was passivated by the addition of Cd(acetate $)_{2}$, leading to a refinement of the optical features. We show that the obtained optical features are comparable to those usually obtained in heterostructures of semiconductors with type I band alignment. The absorption spectra for $3 \mathrm{ML}$ CdSe and $6 \mathrm{ML}$ CdSe NPLs with steps are plotted in Figure 6 (a.) and (b.). These NPLs present multiple excitonic features in absorption corresponding to the hhe and Ih-e transitions in NPLs with various thicknesses. In emission, the NPLs can exhibit 2 to 3 different emission wavelengths centred at the maximum emission for $4 \mathrm{ML}(\approx 510 \mathrm{~nm}), 5 \mathrm{ML}(\approx 550$ $\mathrm{nm}), 6 \mathrm{ML}(\approx 580 \mathrm{~nm}), 7 \mathrm{ML}(\approx 610 \mathrm{~nm})$ and $8 \mathrm{ML}(\approx 625 \mathrm{~nm})$ CdSe NPLs. In any case, the PLE spectra centred on the more red emission fit the absorption spectra. These observations are in agreement with recombination of the exciton in the less confined area of the NPLs, i.e., the thicker part of the homostructure. For example, in Figure 6 (a.), despite absorption spectra dominated by the absorption of $3 \mathrm{ML}$ CdSe NPLs, the emission is redshifted to $507 \mathrm{~nm}$ and $547 \mathrm{~nm}$, which coincides with the emission from 4 and $5 \mathrm{ML}$ CdSe NPLs. Here, the broadening arises from lateral confinement of the exciton when the areas of the islands are small. This is in agreement with a slightly blueshifted emission compared to pure NPLs of the same thickness.

The presence of islands on the $3 \mathrm{MLs}$ induces energy levels from $4 \mathrm{ML}$ and $5 \mathrm{ML}$ in the band gap of the $3 \mathrm{MLCdSe}$ NPLs. The same applies to the $6 \mathrm{ML}$ CdSe NPLs that present energy levels from 7 and $8 \mathrm{ML}$ (see band alignment in Figure 5 (c.)). Currently, there is no proof that all N ML CdSe NPLs present areas of $\mathrm{N}+1$ and $\mathrm{N}+2 \mathrm{ML}$. Therefore, in the mixture, there are most likely N ML CdSe NPLs, N ML CdSe NPLs with a $1 \mathrm{ML}$ island step on one side and N ML CdSe NPLs with $1 \mathrm{ML}$ island on both sides. Therefore, a single NPL can exhibit areas of N MLs, N+1 MLs and N+2 MLs (see Figure 5 (b.)). 
The TEM images (see Figure 6 (d.)) shows contrasted areas in a single NPL, which probably arise from different thicknesses in a single NPL (Here, carboxylates are the only ligands, since $\mathrm{Cd}(\text { acetate })_{2}$ was introduced to stop the reaction).
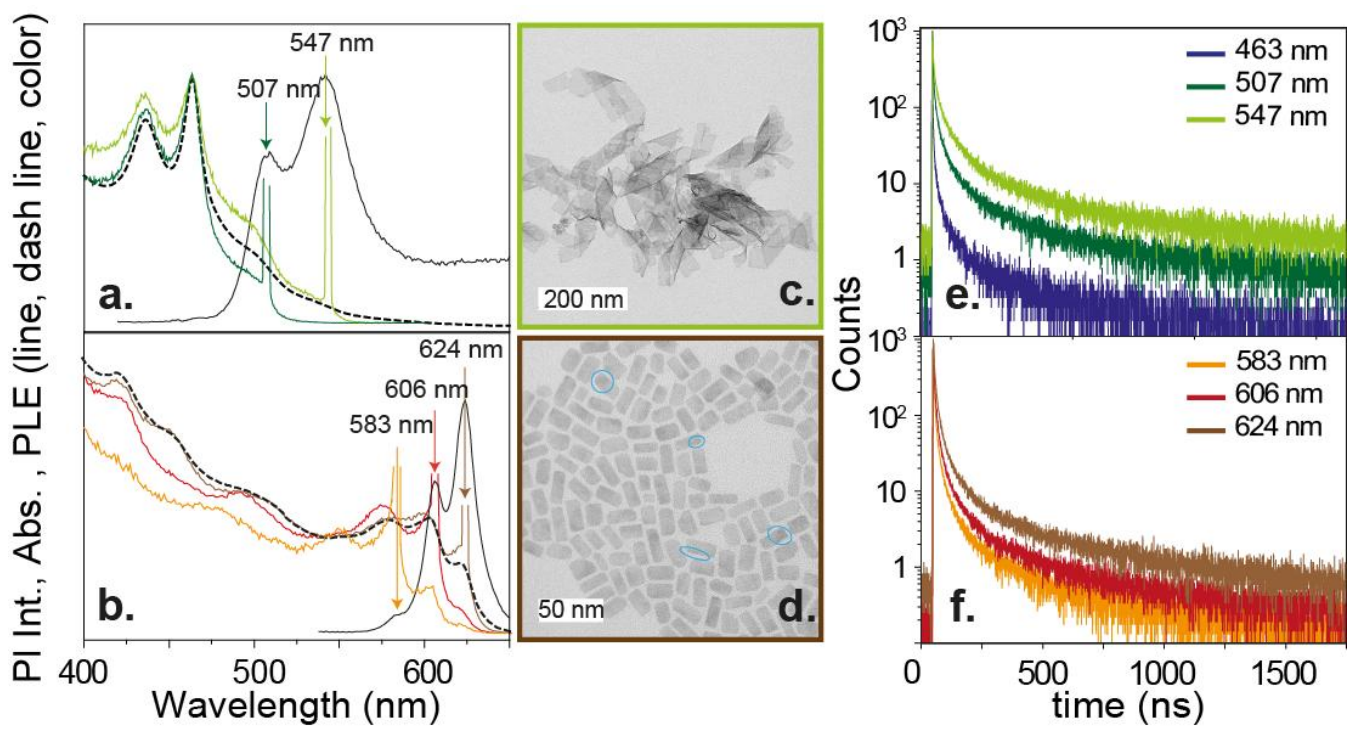

Figure 6: Absorption, photoluminescence intensity and photoluminescence excitation (PLE) spectra for (a.) $3 \mathrm{ML}$ CdSe with steps and (b.) 6 ML CdSe NPLs with steps. (c.) and (d.) TEM images of the corresponding NPLs with steps. The blue ovals indicate contrasting areas, perhaps due to the thicker zone in the NPL. Photoluminescence decays for (e.) 3 ML CdSe with steps and (f.) 6 ML CdSe NPLs with steps.

The fluorescence decay centred at the different emission wavelengths for the two samples is represented in Figure 6 (e.) and (f.). All decays are multiexponential with a long lifetime, which most likely arises from deep traps. It is difficult to give a physical sense to these multiexponential decays; however, it appears that the more red emissions present a slower decay. This observation may have multiple origins. The emission efficiency of bluer emissions is also lower, so the nonradiative decay rate may be stronger in thinner NPLs than in thicker NPLs. Another explanation could be the diffusion of the exciton up to the thicker area of the NPLs due to the energy levels in the gap brought about by the thicker area. Finally, it may also be due to the lateral extension of the steps. Small area islands can be compared to quantum dots in an NPL with an exciton partially confined in the lateral dimension. In that case, the fluorescence lifetime will be longer for spherical nanocrystals compared to nanoplatelets. ${ }^{23}$

Thus, we have synthesized unique homostructures with areas of different confinement levels leading to optical properties that are usually obtained in type I semiconductor. In addition to these unique optical properties, we measured the fluorescence quantum yield for $6 \mathrm{ML}$ CdSe NPLs with steps of $5 \%$, which can be increased to $15 \%$ while using Br-OLAm as ligands. ${ }^{44}$ After the growth of core/crown and core/shell NPLs, it is now possible to add steps to NPLs, enabling the design of new original NPLs with tuned optical features.

\section{CONCLUSION}

To conclude, we have shown that halide ligands can lead to dissolution followed by recrystallization of $\mathrm{CdSe}$ from narrow facets to wide facets. This process is highlighted by an observed 
increase in thickness by 2 monolayers. Therefore, CdSe NPLs of 5 MLs, 7 MLs and 9 MLs can be grown from NPLs of $3 \mathrm{MLs}$, and $6 \mathrm{ML}$ or $8 \mathrm{ML}$ CdSe NPLs can be grown from $4 \mathrm{ML}$ NPLs. This is an original method to grow thick NPLs that is independent of the NPL lateral dimensions and can be applied to other cadmium chalcogenide NPLs, such as CdTe. The activation of the surface reactivity by halides enables the direct growth of metal chalcogenides on $\mathrm{CdSe}$ and $\mathrm{CdTe}$. These methods prevent the use of unstable precursors that are required for the growth of CdSe or CdTe by c-ALD. Finally, we also demonstrate the synthesis of a unique type of homostructure, where variations in thickness in a unique NPL lead to recombination of the exciton in the thicker parts. 


\section{METHODS}

\section{Chemicals}

Octadecene (ODE) (Sigma-Aldrich, 90\%), Cadmium Acetate anhydrous ( $\left.\mathrm{Cd}(\mathrm{Ac})_{2}\right)$ (Sigma-Aldrich, 99.995\%), Cadmium Oxide (CdO) (Strem 99.99\%), Myristic Acid (Sigma-Aldrich 99\%), Propionic Acid (Sigma-Aldrich 99\%), Selenium powder (Se) (Strem chemicals 99.99\%), Tellurium powder (Te) (SigmaAldrich 99.99\%), Oleic Acid (OA) (Sigma-Aldrich 90\%), Trioctylphosphine (TOP) (Cytec, Solvay), Tributylphsopine (TBP) (Cytec, Solvay), Cadmium Chloride hydrate $\left(\mathrm{CdCl}_{2} \cdot \mathrm{xH}_{2} \mathrm{O}\right)$ (Sigma-Aldrich 99.995\%), Cadmium Bromide tetrahydrate $\left(\mathrm{CdBr}_{2} .4 \mathrm{H}_{2} \mathrm{O}\right)$ (Sigma-Aldrich 98\%), Cadmium lodide $\left(\mathrm{CdI}_{2}\right)$ (Sigma-Aldrich 99\%), Zinc Chloride) ( $\mathrm{ZnCl}_{2}$ ) (Sigma-Aldrich 98\%), Toluene (VWR 99.5\%), n-Hexane (VWR 99\%), Ethanol (VWR 96\%), Acetone (VWR 99\%).

\section{Precursor Preparation}

Cadmium Myristate $\mathrm{Cd}(\mathrm{Myr})_{2}$. In a $50 \mathrm{~mL}$ three-neck flask, $2.56 \mathrm{~g}$ of $\mathrm{CdO}(20 \mathrm{mmol})$ and $11 \mathrm{~g}$ of myristic acid $(50 \mathrm{mmol})$ were mixed and degassed for $30 \mathrm{~min}$ at $80^{\circ} \mathrm{C}$. Then, under argon flow, the temperature was set to $200^{\circ} \mathrm{C}$ until the solution became colourless. At $60^{\circ} \mathrm{C}, 30 \mathrm{~mL}$ of methanol was added to solubilize the excess myristic acid. Cadmium myristate was precipitated by using a centrifuge tube with methanol. The washing process was repeated at least three times. The final powder was dried overnight under vacuum at $70^{\circ} \mathrm{C}$.

Cadmium Propionate Cd(Prop) 2 . In a $100 \mathrm{~mL}$ three-neck flask, $5.18 \mathrm{~g}$ of CdO $(40.3 \mathrm{mmol})$ and $50 \mathrm{~mL}$ of propionic acid were mixed and heated at $70^{\circ} \mathrm{C}$ for $1 \mathrm{~h}$ under argon. When the entire solid was dissolved and a colourless solution was obtained, heating was stopped, and acetone was added to precipitate cadmium propionate. The powder was centrifuged and washed at least 3 times with acetone and then set under vacuum overnight.

Cadmium Oleate $\mathrm{Cd}(\mathrm{OA})_{2}$ at $0.5 \mathrm{M}$. In a $100 \mathrm{~mL}$ three-neck flask, $30 \mathrm{~mL}$ of oleic acid and $1.92 \mathrm{~g}$ of $\mathrm{CdO}$ were heated to $200^{\circ} \mathrm{C}$ under argon flow for $1 \mathrm{~h}$. The temperature was then decreased to $60^{\circ} \mathrm{C}$, and the solution degassed for $1 \mathrm{~h}$.

$C d X_{2}(X=C l, B r, l) 0.5 \mathrm{M}$ solution in TBP or mix of TBP/TOP. First, $0.32 \mathrm{mmol}$ of $\mathrm{CdX} \mathrm{X}_{2}$ was added to 300 $\mu \mathrm{L}$ of TBP. The solution was sonicated and heated with a heat gun until full dissolution of the solid precursor. Then, the solution was diluted by adding $300 \mu \mathrm{L}$ of TBP or $300 \mu \mathrm{L}$ of TOP. The solution remained clear over several days.

\section{Nanoplatelets synthesis}

CdSe $3 \mathrm{MLs} 463 \mathrm{~nm}$. In a $50 \mathrm{~mL}$ three-neck flask, $240 \mathrm{mg}$ of $\mathrm{Cd}(\mathrm{Ac})_{2}$ and $15 \mathrm{~mL}$ of ODE were mixed and degassed at $60^{\circ} \mathrm{C}$ for $30 \mathrm{~min}$. Under argon flow, the temperature was set to $190^{\circ} \mathrm{C}$, and then a mixture of $600 \mu \mathrm{L}$ of TOPSe $1 \mathrm{M}, 200 \mu \mathrm{L}$ of OA and $5 \mathrm{~mL}$ of ODE was added dropwise at a rate of 4 $\mathrm{mL} / \mathrm{h}$. The mixture was then cooled down to room temperature. The NPLs were centrifuged twice with $15 \mathrm{~mL}$ of hexane and $15 \mathrm{~mL}$ of ethanol and then redispersed in $10 \mathrm{~mL}$ of hexane.

CdSe $4 \mathrm{MLs} 510 \mathrm{~nm}$. In a $50 \mathrm{~mL}$ three-neck flask, $24 \mathrm{mg}$ of Se, $340 \mathrm{mg}$ of Cd(Myr $)_{2}$ and $30 \mathrm{~mL}$ of ODE were mixed and degassed at room temperature for $1 \mathrm{~h}$. Under argon flow, the temperature was set to $230^{\circ} \mathrm{C}$. At $205^{\circ} \mathrm{C}$, the solution turned orange, and $110 \mathrm{mg}$ of $\mathrm{Cd}(\mathrm{Ac})_{2}$ was added to the mixture. 
After $10 \mathrm{~min}$ at $230^{\circ} \mathrm{C}$, the mixture was cooled, and $500 \mu \mathrm{L}$ of OA was added. The NPLs were centrifuged twice with $10 \mathrm{~mL}$ of hexane and $20 \mathrm{~mL}$ of ethanol and then redispersed in $10 \mathrm{~mL}$ of hexane.

CdTe $3 \mathrm{MLs} 500 \mathrm{~nm} \cdot{ }^{49} \mathrm{In}$ a $100 \mathrm{~mL}$ three-neck flask, $390 \mathrm{mg}$ of Cd(Prop) $2,240 \mu \mathrm{L}$ of OA and $30 \mathrm{~mL}$ of ODE were mixed and degassed at $90^{\circ} \mathrm{C}$ for $1 \mathrm{~h}$. Under argon flow, the temperature was set to $210^{\circ} \mathrm{C}$. Then, a mixture of $300 \mu \mathrm{L}$ of TOPTe $1 \mathrm{M}$ and $1.5 \mathrm{~mL}$ of ODE was added to the mixture. After $30 \mathrm{~min}$ at $210^{\circ} \mathrm{C}$, the mixture was cooled, and $1.7 \mathrm{~mL}$ was added. The NPLs were centrifuged twice with $15 \mathrm{~mL}$ of hexane and $20 \mathrm{~mL}$ of ethanol and then redispersed in $30 \mathrm{~mL}$ of hexane.

\section{Dissolution/recrystallization growth}

CdSe $3 \mathrm{MLs} 463 \mathrm{~nm}$ growth. In a three-neck flask, $500 \mu \mathrm{L}$ of NPLs, $10 \mu \mathrm{L}$ of OA and $3 \mathrm{~mL}$ of ODE were mixed and degassed at room temperature for $15 \mathrm{~min}$. Under argon flow, the temperature was set to $150^{\circ} \mathrm{C}$, and then $40 \mu \mathrm{L}$ of a chloride-based growth mixture was added to the solution. The kinetics of the reaction were followed up by measuring the absorption spectra. When the first excitonic peak reached the desired wavelength ( $565 \mathrm{~nm}, 620 \mathrm{~nm}$ or $650 \mathrm{~nm}$ for $5 \mathrm{MLs}, 7 \mathrm{MLs}$ or $9 \mathrm{MLs}$, respectively), the reaction was quenched by adding $20 \mathrm{mg}$ of $\mathrm{Cd}$ (acetate) ${ }_{2}$ and $\mathrm{nH}_{2} \mathrm{O}$ powder. After $20 \mathrm{~min}$, the temperature was decreased to room temperature. The NPLs were precipitated twice with a toluene/ethanol (2/3) mixture for $5 \mathrm{~min}$ at $6000 \mathrm{rpm}$ and redispersed in $5 \mathrm{~mL}$ of toluene.

CdSe $4 \mathrm{MLs} 510 \mathrm{~nm}$. In a three-neck flask, $1.5 \mathrm{~mL}$ of NPLs, $30 \mu \mathrm{L}$ of OA and $3 \mathrm{~mL}$ of ODE were mixed and degassed at room temperature for $15 \mathrm{~min}$. Under argon flow, the temperature was set to $150^{\circ} \mathrm{C}$, and $40 \mu \mathrm{L}$ of a chloride-based growth mixture was added to the solution. The kinetics of the reaction were followed up by measuring the absorption spectra. When the first excitonic peak reached the desired wavelength ( $585 \mathrm{~nm}$ or $635 \mathrm{~nm}$ for $6 \mathrm{MLs}$ or $8 \mathrm{MLs}$, respectively), the reaction was quenched by adding $20 \mathrm{mg}$ of $\mathrm{Cd}$ (acetate) ${ }_{2}$ and $\mathrm{nH}_{2} \mathrm{O}$ powder. After $20 \mathrm{~min}$, the temperature was cooled to room temperature. The NPLs were precipitated twice with a toluene/ethanol (2/3) mixture for 5 min at $6000 \mathrm{rpm}$ and redispersed in $5 \mathrm{~mL}$ of toluene.

CdTe $3 \mathrm{MLs} 500 \mathrm{~nm}$. In a three-neck flask, $3 \mathrm{~mL}$ of NPLs, $10 \mu \mathrm{L}$ of OA, $40 \mu \mathrm{L}$ of a bromide-based growth mixture and $3 \mathrm{~mL}$ of ODE were mixed and degassed at room temperature for $15 \mathrm{~min}$. Under argon flow, the temperature was set to $150^{\circ} \mathrm{C}$, and the kinetics of the reaction were determined by absorption spectroscopy. During the reaction, the temperature was increased up to $170^{\circ} \mathrm{C}$ (or $190^{\circ} \mathrm{C}$ ), and when the first excitonic peak reached $580 \mathrm{~nm}$ (or $630 \mathrm{~nm}$ ), the reaction was quenched by adding $20 \mathrm{mg}$ of $\mathrm{Cd}(\text { acetate) })_{2}$ and $\mathrm{nH}_{2} \mathrm{O}$ powder. The temperature was increased up to $180^{\circ} \mathrm{C}$ (or $200^{\circ} \mathrm{C}$ ) for $30 \mathrm{~min}$ and then decreased when the excitonic peak was stabilized at $602 \mathrm{~nm}$ (or $633 \mathrm{~nm}$ ) for $5 \mathrm{MLs}$ (or $7 \mathrm{MLs}$ ). The NPLs were then precipitated twice with a toluene/ethanol (2/3) mixture for $5 \mathrm{~min}$ at $6000 \mathrm{rpm}$, redispersed in $5 \mathrm{~mL}$ of toluene and kept in a glovebox.

The growth of NPLs with steps was obtained when $\mathrm{Cd}$ (acetate) ${ }_{2}, \mathrm{nH}_{2} \mathrm{O}$ was added before the completion of a full layer.

\section{Growth of homostructures and heterostructures}

Core/shell on large $3 \mathrm{ML}$ CdSe NPLs. In a three-neck flask, $500 \mu \mathrm{L}$ of NPLs, $10 \mu \mathrm{L}$ of OA and $3 \mathrm{~mL}$ of ODE were mixed and degassed at room temperature for $15 \mathrm{~min}$. Under argon flow, the temperature was set to $160^{\circ} \mathrm{C}$, and then $45 \mu \mathrm{L}$ of a mixture of $22.5 \mu \mathrm{L}$ of TOPSe at $0.5 \mathrm{M}$ and $22.5 \mu \mathrm{L}$ of a solution 
of $\mathrm{CdCl}_{2}$ in TBP at $0.5 \mathrm{M}$ was injected. After 10 to $15 \mathrm{~min}, 20 \mathrm{mg}$ of $\mathrm{Cd}(\text { acetate })_{2}$ and $\mathrm{nH}_{2} \mathrm{O}$ were introduced into the reaction mixture and allowed to stand for $10 \mathrm{~min}$. The temperature was raised to $180^{\circ} \mathrm{C}$ to favour surface rearrangements. After an additional $5 \mathrm{~min}$, the mixture was cooled, and the NPLs were centrifuged with ethanol and dispersed in hexane. This cycle was repeated to grow thicker NPLs. The injected solution can be replaced by a mix of $\mathrm{CdCl}_{2} / \mathrm{TBP}$ and TOPTe to grow CdTe shells or a mix of $\mathrm{ZnCl}_{2} / \mathrm{TBP}$ and TOPSe to grow $\mathrm{ZnSe}$ shells.

\section{ASSOCIATED CONTENT}

\section{Supporting information}

The Supporting Information is available free of charge at

Supporting Information includes additional synthesis showing the effect of OA, halides, and EDX measurements.

\section{ACKNOWLEDGMENTS}

SI thanks the support from an ERC starting grant Ne2DeM (grant $n^{\circ}$ 853049). EL thanks the support from an ERC starting grant blackQD (grant $n^{\circ} 756225$ ).

\section{References}

(1) Nasilowski, M.; Mahler, B.; Lhuillier, E.; Ithurria, S.; Dubertret, B. Two-Dimensional Colloidal Nanocrystals. Chem. Rev. 2016, 116 (18), 10934-10982.

(2) Benchamekh, R.; Gippius, N. A.; Even, J.; Nestoklon, M. O.; Jancu, J. M.; Ithurria, S.; Dubertret, B.; Efros, A. L.; Voisin, P. Tight-Binding Calculations of Image-Charge Effects in Colloidal Nanoscale Platelets of CdSe. Phys. Rev. B 2014, 89 (3), 035307.

(3) Scott, R.; Achtstein, A. W.; Prudnikau, A. V.; Antanovich, A.; Siebbeles, L. D. a; Artemyev, M.; Woggon, U. Time-Resolved Stark Spectroscopy in CdSe Nanoplatelets: Exciton Binding Energy, Polarizability, and Field-Dependent Radiative Rates. Nano Lett. 2016, 16 (10), 6576-6583.

(4) Tessier, M. D.; Javaux, C.; Maksimovic, I.; Loriette, V.; Dubertret, B. Spectroscopy of Single CdSe. ACS Nano 2012, 6 (8), 6751-6758.

(5) Joo, J.; Son, J. S.; Kwon, S. G.; Yu, J. H.; Hyeon, T. Low-Temperature Solution-Phase Synthesis of Quantum Well Structured CdSe Nanoribbons. J. Am. Chem. Soc. 2006, 128 (17), 5632-5633.

(6) Wang, F.; Wang, Y.; Liu, Y.-H.; Morrison, P. J.; Loomis, R. a; Buhro, W. E. Two-Dimensional Semiconductor Nanocrystals: Properties, Templated Formation, and Magic-Size Nanocluster Intermediates. Acc. Chem. Res. 2015, 48 (1), 13-21.

(7) Bekenstein, Y.; Koscher, B. A.; Eaton, S. W.; Yang, P.; Alivisatos, A. P. Highly Luminescent Colloidal Nanoplates of Perovskite Cesium Lead Halide and Their Oriented Assemblies. J. Am. Chem. Soc. 2015, 137 (51), 16008-16011. 
(8) Schliehe, C.; Juarez, B. H.; Pelletier, M.; Jander, S.; Greshnykh, D.; Nagel, M.; Meyer, A.; Foerster, S.; Kornowski, A.; Klinke, C.; Weller, H. Ultrathin PbS Sheets by Two-Dimensional Oriented Attachment. Science 2010, 329 (5991), 550-553.

(9) Khan, A. H.; Brescia, R.; Polovitsyn, A.; Angeloni, I.; Martín-García, B.; Moreels, I. Near-Infrared Emitting Colloidal PbS Nanoplatelets: Lateral Size Control and Optical Spectroscopy. Chem. Mater. 2017, 29 (7), 2883-2889.

(10) Izquierdo, E.; Robin, A.; Keuleyan, S.; Lequeux, N.; Lhuillier, E.; Ithurria, S. Strongly Confined HgTe 2D Nanoplatelets as Narrow near Infrared Emitters. J. Am. Chem. Soc. 2016, 138 (33), 10496-10501.

(11) Sharma, M.; Olutas, M.; Yeltik, A.; Kelestemur, Y.; Sharma, A.; Delikanli, S.; Guzelturk, B.; Gungor, K.; McBride, J. R.; Demir, H. V. Understanding the Journey of Dopant Copper lons in Atomically Flat Colloidal Nanocrystals of CdSe Nanoplatelets Using Partial Cation Exchange Reactions. Chem. Mater. 2018, 30 (10), 3265-3275.

(12) Khan, A. H.; Pinchetti, V.; Tanghe, I.; Dang, Z.; Martín-García, B.; Hens, Z.; Van Thourhout, D.; Geiregat, P.; Brovelli, S.; Moreels, I. Tunable and Efficient Red to Near-Infrared Photoluminescence by Synergistic Exploitation of Core and Surface Silver Doping of CdSe Nanoplatelets. Chem. Mater. 2019, 31 (4), 1450-1459.

(13) Dufour, M.; Izquierdo, E.; Livache, C.; Martinez, B.; Silly, M. G.; Pons, T.; Lhuillier, E.; Delerue, C.; Ithurria, S. Doping as a Strategy to Tune Color of 2D Colloidal Nanoplatelets. ACS Appl. Mater. Interfaces 2019, 11 (10), 10128-10134.

(14) Prudnikau, A.; Chuvilin, A.; Artemyev, M. CdSe-CdS Nanoheteroplatelets with Efficient Photoexcitation of Central CdSe Region through Epitaxially Grown CdS Wings. J. Am. Chem. Soc. 2013, 135 (39), 14476-14479.

(15) Tessier, M. D.; Spinicelli, P.; Dupont, D.; Patriarche, G.; Ithurria, S.; Dubertret, B. Efficient Exciton Concentrators Built from Colloidal Core/Crown CdSe/CdS Semiconductor Nanoplatelets. Nano Lett. 2014, 14 (1), 207-213.

(16) Pedetti, S.; Ithurria, S.; Heuclin, H.; Patriarche, G.; Dubertret, B. Type-II CdSe/CdTe Core/Crown Semiconductor Nanoplatelets. J. Am. Chem. Soc. 2014, 136 (46), 16430-16438.

(17) Kelestemur, Y.; Guzelturk, B.; Erdem, O.; Olutas, M.; Erdem, T.; Usanmaz, C. F.; Gungor, K.; Demir, H. V. CdSe/CdSe1-XTex Core/Crown Heteronanoplatelets: Tuning the Excitonic Properties without Changing the Thickness. J. Phys. Chem. C 2017, 121 (8), 4650-4658.

(18) Dufour, M.; Steinmetz, V.; Izquierdo, E.; Pons, T.; Lequeux, N.; Lhuillier, E.; Legrand, L.; Chamarro, M.; Barisien, T.; Ithurria, S. Engineering Bicolor Emission in 2D Core/Crown CdSe/CdSe1-XTex Nanoplatelet Heterostructures Using Band-Offset Tuning. J. Phys. Chem. C 2017, 121 (39), 24816-24823.

(19) Khan, A. H.; Bertrand, G. H. V.; Teitelboim, A.; Sekhar M, C.; Polovitsyn, A.; Brescia, R.; Planelles, J.; Climente, J. I.; Oron, D.; Moreels, I. CdSe/CdS/CdTe Core/Barrier/Crown Nanoplatelets: Synthesis, Optoelectronic Properties, and Multiphoton Fluorescence Upconversion. ACS Nano 2020, 14 (4), 4206-4215.

(20) Guzelturk, B.; Kelestemur, Y.; Olutas, M.; Delikanli, S.; Demir, H. V. Amplified Spontaneous Emission and Lasing in Colloidal Nanoplatelets. ACS Nano 2014, 8 (7), 6599-6605.

(21) Gao, Y.; Li, M.; Delikanli, S.; Zheng, H.; Liu, B.; Dang, C.; Sum, T. C.; Demir, H. V. Low-Threshold 
Lasing from Colloidal CdSe/CdSeTe Core/Alloyed-Crown Type-II Heteronanoplatelets. Nanoscale 2018, 10 (20), 9466-9475.

(22) Ithurria, S.; Bousquet, G.; Dubertret, B. Continuous Transition from 3D to 1D Confinement Observed during the Formation of CdSe Nanoplatelets. J. Am. Chem. Soc. 2011, 133 (9), 30703077.

(23) Ithurria, S.; Tessier, M. D.; Mahler, B.; Lobo, R. P. S. M.; Dubertret, B.; Efros, A. L. Colloidal Nanoplatelets with Two-Dimensional Electronic Structure. Nature Materials. 2011, pp 936941.

(24) Ithurria, S.; Talapin, D. V. Colloidal Atomic Layer Deposition (c-ALD) Using Self-Limiting Reactions at Nanocrystal Surface Coupled to Phase Transfer between Polar and Nonpolar Media. J. Am. Chem. Soc. 2012, 134 (45), 18585-18590.

(25) Hazarika, A.; Fedin, I.; Hong, L.; Guo, J.; Srivastava, V.; Cho, W.; Coropceanu, I.; Portner, J.; Diroll, B. T.; Philbin, J. P.; Rabani, E.; Klie, R.; Talapin, D. V. Colloidal Atomic Layer Deposition with Stationary Reactant Phases Enables Precise Synthesis of "Digital" II-VI NanoHeterostructures with Exquisite Control of Confinement and Strain. J. Am. Chem. Soc. 2019, 141 (34), 13487-13496.

(26) Mahler, B.; Nadal, B.; Bouet, C.; Patriarche, G.; Dubertret, B. Core/Shell Colloidal Semiconductor Nanoplatelets. J. Am. Chem. Soc. 2012, 134 (45), 18591-18598.

(27) Rossinelli, A. A.; Riedinger, A.; Marqués-Gallego, P.; Knüsel, P. N.; Antolinez, F. V.; Norris, D. J. High-Temperature Growth of Thick-Shell CdSe/CdS Core/Shell Nanoplatelets. Chem. Commun. 2017, 53 (71), 9938-9941.

(28) Altintas, Y.; Gungor, K.; Gao, Y.; Sak, M.; Quliyeva, U.; Bappi, G.; Mutlugun, E.; Sargent, E. H.; Demir, H. V. Giant Alloyed Hot Injection Shells Enable Ultralow Optical Gain Threshold in Colloidal Quantum Wells. ACS Nano 2019, 13 (9), 10662-10670.

(29) Feng, F.; Nguyen, L. T.; Nasilowski, M.; Nadal, B.; Dubertret, B.; Maître, A.; Coolen, L. Probing the Fluorescence Dipoles of Single Cubic CdSe/CdS Nanoplatelets with Vertical or Horizontal Orientations. ACS Photonics 2018, 5 (5), 1994-1999.

(30) Liu, B.; Altintas, Y.; Wang, L.; Shendre, S.; Sharma, M.; Sun, H.; Mutlugun, E.; Demir, H. V. Record High External Quantum Efficiency of 19.2\% Achieved in Light-Emitting Diodes of Colloidal Quantum Wells Enabled by Hot-Injection Shell Growth. Adv. Mater. 2019, 32 (8), 1905824.

(31) Rossinelli, A. A.; Rojo, H.; Mule, A. S.; Aellen, M.; Cocina, A.; De Leo, E.; Schäublin, R.; Norris, D. J. Compositional Grading for Efficient and Narrowband Emission in CdSe-Based Core/Shell Nanoplatelets. Chem. Mater. 2019, 31 (22), 9567-9578.

(32) Christodoulou, S.; Climente, J. I.; Planelles, J.; Brescia, R.; Prato, M.; Martin-Garcia, B.; Khan, A. H.; Moreels, I. Chloride-Induced Thickness Control in CdSe Nanoplatelets. Nano Lett. 2018, 18 (10), 6248-6254.

(33) Cho, W.; Kim, S.; Coropceanu, I.; Srivastava, V.; Diroll, B. T.; Hazarika, A.; Fedin, I.; Galli, G.; Schaller, R. D.; Talapin, D. V. Direct Synthesis of Six-Monolayer (1.9 Nm) Thick Zinc-Blende CdSe Nanoplatelets Emitting at 585 Nm. Chem. Mater. 2018, 30, 6957-6960.

(34) Meerbach, C.; Wu, C.; Erwin, S. C.; Dang, Z.; Prudnikau, A.; Lesnyak, V. Halide-Assisted Synthesis of Cadmium Chalcogenide Nanoplatelets. Chem. Mater. 2020, 32 (1), 566-574. 
(35) Riedinger, A.; Ott, F. D.; Mule, A.; Mazzotti, S.; Knüsel, P. N.; Kress, S. J. P.; Prins, F.; Erwin, S. C.; Norris, D. J. An Intrinsic Growth Instability in Isotropic Materials Leads to Quasi-TwoDimensional Nanoplatelets. Nat. Mater. 2017, 16 (7), 743-748.

(36) Ghosh, S.; Manna, L. The Many "Facets" of Halide lons in the Chemistry of Colloidal Inorganic Nanocrystals. Chem. Rev. 2018, 118 (16), 7804-7864.

(37) Gerdes, F.; Navío, C.; Juárez, B. H.; Klinke, C. Size, Shape, and Phase Control in Ultrathin CdSe Nanosheets. Nano Lett. 2017, 17 (7), 4165-4171.

(38) Goubet, N.; Jagtap, A.; Livache, C.; Martinez, B.; Portalès, H.; Xu, X. Z.; Lobo, R. P. S. M.; Dubertret, B.; Lhuillier, E. Terahertz HgTe Nanocrystals: Beyond Confinement. J. Am. Chem. Soc. 2018, 140 (15), 5033-5036.

(39) Tessier, M. D.; Dupont, D.; De Nolf, K.; De Roo, J.; Hens, Z. Economic and Size-Tunable Synthesis of InP/ZnE (E = S, Se) Colloidal Quantum Dots. Chem. Mater. 2015, 27 (13), 48934898.

(40) Smith, A. M.; Mohs, A. M.; Nie, S. Tuning the Optical and Electronic Properties of Colloidal Nanocrystals by Lattice Strain. Nat. Nanotechnol. 2009, 4 (1), 56-63.

(41) Chu, A.; Livache, C.; Ithurria, S.; Lhuillier, E. Electronic Structure Robustness and Design Rules for 2D Colloidal Heterostructures Electronic Structure Robustness and Design Rules for 2D Colloidal Heterostructures. J. Appl. Phys. 2018, 123 (035701), 1-6.

(42) Goel, R. G.; Ogini, W. O. Preparation, Characterization, and Spectral Studies of Neutral TriTert-Butylphosphine Complexes of Zinc(II) and Cadmium(II). Inorg. Chem. 1977, 16 (8), 19681972.

(43) Goel, R. G.; Henry, W. P.; Jha, N. K. Reinvestigation of Tricyclohexylphosphine Complexes of Zinc(II) and Cadmium(II) Halides. Preparation, Characterization, and Phosphorus-31 Nuclear Magnetic Resonance and Vibrational Spectroscopic Studies. Inorg. Chem. 1982, 21 (7), 25512555.

(44) Dufour, M.; Qu, J.; Greboval, C.; Méthivier, C.; Ithurria, S. Halide Ligands to Release Strain in Cadmium Chalcogenide Nanoplatelets and Achieve High Brightness. ACS Nano 2019, 13 (5), 5326-5334.

(45) Ott, F. D.; Riedinger, A.; Ochsenbein, D. R.; Knüsel, P. N.; Erwin, S. C.; Mazzotti, M.; Norris, D. J. Ripening of Semiconductor Nanoplatelets. Nano Lett. 2017, 17 (11), 6870-6877.

(46) Singh, S.; Tomar, R.; Ten Brinck, S.; De Roo, J.; Geiregat, P.; Martins, J. C.; Infante, I.; Hens, Z. Colloidal CdSe Nanoplatelets, A Model for Surface Chemistry/Optoelectronic Property Relations in Semiconductor Nanocrystals. J. Am. Chem. Soc. 2018, 140 (41), 13292-13300.

(47) Eyring, H. The Activated Complex in Chemical Reactions. J. Chem. Phys. 1935, 3 (2), 63-71.

(48) Laidler, K. J.; King, M. C. The Development of Transition-State Theory. J. Phys. Chem. 1983, 87 (15), 2657-2664.

(49) Pedetti, S.; Nadal, B.; Lhuillier, E.; Mahler, B.; Bouet, C.; Abécassis, B.; Xu, X.; Dubertret, B. Optimized Synthesis of CdTe Nanoplatelets and Photoresponse of CdTe Nanoplatelets Films. Chem. Mater. 2013, 25 (12), 2455-2462. 


\section{Table of Contents graphic:}

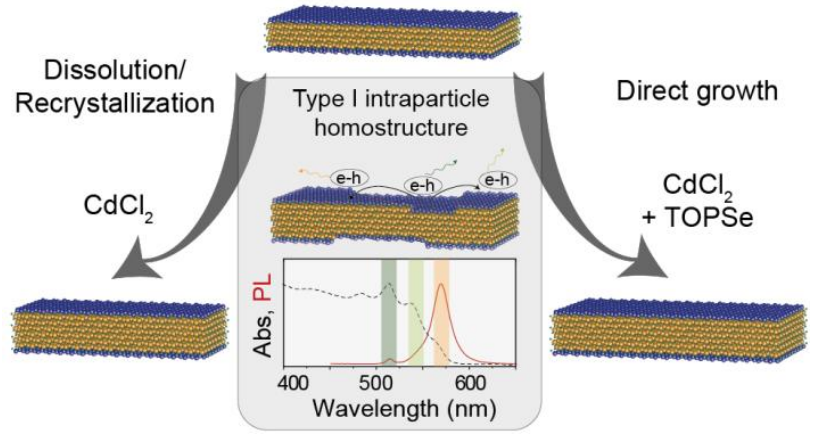

